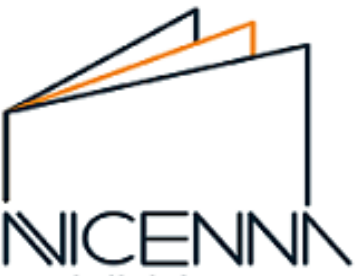

publishing $\mathrm{co}$.

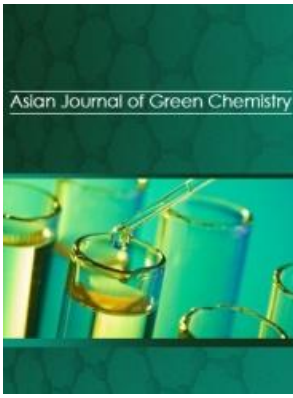

Original Research article

\title{
Sulfonylbis(1,4-phenylene)bissulfamic acid (SPSA): Introduction of an efficient and reusable catalyst for the synthesis of bis(indolyl)methanes
}

\author{
Masoud Mohammadi Zeydi*, Nosrat O Allah Mahmoodi, Ghorban Ardeshiri Terogeni \\ Department of Organic Chemistry, Faculty of Sciences, University of Guilan, Rasht, Iran
}

\section{ARTICLE INFORMATION}

Received: 15 August 2017

Received in revised: 25 August 2017

Accepted: 25 August 2017

Available online: 31 August 2017

DOI:10.22631/ajgc.2017.95432.1013

\section{KEYWORDS}

Sulfonylbis(1,4-phenylene)bissulfamic acid (SPSA)

Bis-indolyl methanes

Aldehyde

Indoles

Multi-component reactions

\section{ABSTRACT}

A simple, efficient and convenient route is described for the synthesis of bisindolyl methanes by using recyclable catalyst sulfonylbis(1,4phenylene)bissulfamic acid (SPSA). In this procedure, we synthesize a bis-indolyl methane derivative via the three component reactions of two equivalent indoles with one equivalent of various aromatic aldehydes in the presence of $10 \mathrm{~mol} \% \mathrm{SBSA}$ as a heterogeneous catalyst under solvent-free conditions at $110{ }^{\circ} \mathrm{C}$ for the convinced reaction times (30-60 min). The advantages of this protocol towards the synthesis of bis-indolyl methane derivatives are: I) use of solvent-free conditions, II) inexpensive catalyst, III) using commercially available precursors, d) reusability of SBSA up to several cycles without much loss in reactivity, IV) simple work-up, V) high yields of pure products, VI) short reaction times. The structure of all bis(indolyl)methane derivatives were confirmed by melting point, FT-IR, ${ }^{1} \mathrm{H}$ NMR spectra and were compared with reliable references. 


\section{Graphical Abstract}
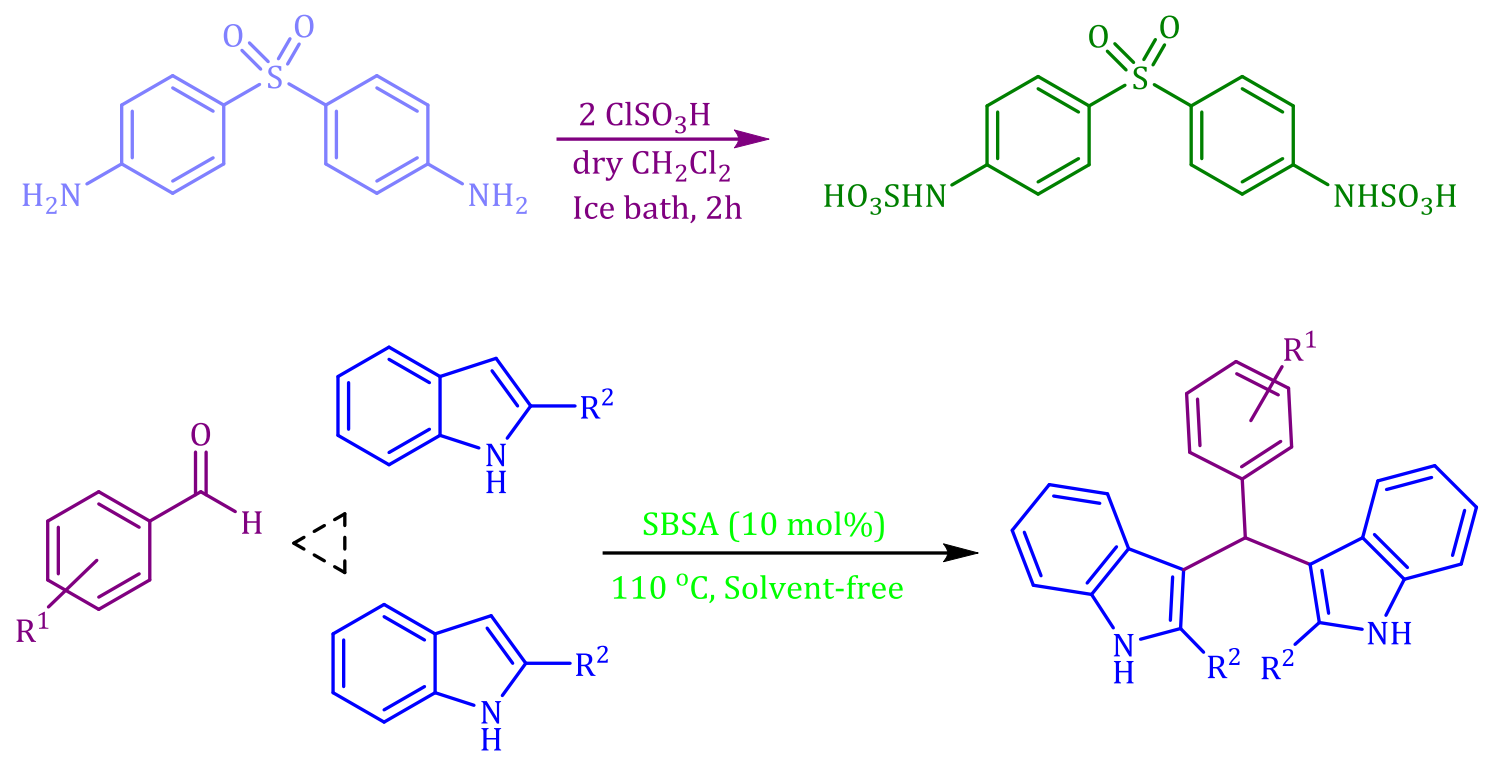

\section{Introduction}

Recently, multi-component reactions (MCRs) have been widely paid attention by synthetic organic chemist from all over the world due to their efficacy for the building of heterocyclic complex molecules with diverse ranges of complexity in a single synthetic step from available starting molecules. These reactions represent very strong chemical method from both synthetic and economical points of view and time-efficient reactions are one of the most influential methods in industrial chemistry, green chemistry and the novel drug discovery process. Hence, the discovery of novel multicomponent is an interesting subject for organic chemistry researchers $[1,2]$.

The most important goals in the designing of catalysts, in terms of green chemistry, is to expand environmentally benign, economical, clean, practical, and efficient methods for catalyst separation and recycling. A convenient way of achieving these goals is the use of heterogeneous catalyst system for various organic reactions [3-6]. A heterogeneous catalyst system provides more filtration as it avoids catalyst loss. We have recently reported sulfonylbis(1,4phenylene)bissulfamic acid (SPSA) [7], as an effective heterogeneous acidic catalyst for the preparation of chromene derivatives. simple work-up procedures, ease of preparation, and improved product yields, facile purification, shorter reaction times, mild reaction conditions, more stability, reusability, low toxicity and ease of handling of the catalyst are the main superiorities of SPSA.

Bis(indolyl)methanes, indole and its derivatives are a valuable group of nitrogen heterocyclic compound, which known as an kind of natural and synthetic intermediates in pharmaceutical 
industry [8]. Bis(indolyl)methanes possessing diverse pharmacological activities such as anticancer [9], antimicrobial [10], anti-oxidant [11], and anti-inflammatory [12] activities; also, these types of compounds can aid the inhibition cancer cell growth via the induction of metastasis and apoptosis [13]. The main approach for the direct synthesis of this class of molecules is based on the electrophilic substitution of indole with carbonyl compounds in the presence of acidic catalysts such as silica sulfuric acid (SSA) [14], tetrabutylammo-nium tribromide [15], metal hydrogen sulfates [16], sulfated zirconia [17], montmorillonite clay K-10 [18], sodium dodecyl sulfate [19], trichloro-1,3,5-triazine [20], Zeolites [21], $\mathrm{AlPW}_{12} \mathrm{O}_{40}$ [22], $\mathrm{InCl}_{3}$ [23], $\mathrm{H}_{3} \mathrm{PW}_{12} \mathrm{O}_{40}$ [24], $\mathrm{TiO}_{2}$ [25], $\mathrm{LiClO}_{4}$ [26], $\mathrm{ZrOCl}_{2} / \mathrm{SiO}_{2}$ [27], $\mathrm{NaBF}_{4}$ [28], $\mathrm{HClO}_{4}-\mathrm{SiO}_{2}$ [29], NBS [30], and $\mathrm{Bi}\left(\mathrm{NO}_{3}\right)_{3} .5 \mathrm{H}_{2} \mathrm{O}$ [31]. Of course, most of the reported methods involve toxic solvent and metal ions, cumbersome work-up procedures and high cost.

In this research we wish to report the applicability of sulfonylbis(1,4-phenylene)bissulfamic acid (SPSA) in the promotion of the preparation of bis(indolyl)methanes through the electrophilic substitution of indole derivatives with various aldehydes.

\section{Experimental}

Matreials and methodes

All chemicals were purchased from Fluka and Merck chemical companies. Melting points were recorded by using capillary tubes on an electrothermal 9100 apparatus and are uncorrected. The IR and FT-IR spectra were obtained using a 4300 Shimadzu spectrophotometer as KBr disks. ${ }^{1} \mathrm{H}$ NMR spectra were recorded on a DPX $400 \mathrm{MHz}$ spectrometer in $\mathrm{CDCl}_{3}$ as the solvent relative to TMS. All yields refer to the isolated products and the known products were characterized by their physical constants and comparison with valid samples.

General procedure for the preparation of sulfonylbis(1,4-phenylene)bissulfamic acid (SPSA)

A $15 \mathrm{~mL}$ suction flask charged with a solution of 4,4'-sulfonyldianiline (10 mmol, $2.843 \mathrm{~g}$ ) in dry $\mathrm{CH}_{2} \mathrm{Cl}_{2}(5 \mathrm{~mL})$ was equipped with a constant pressure-dropping funnel containing $1.165 \mathrm{~g}$ chlorosulfonic acid (10 mmol) and a gas inlet tube for conducting $\mathrm{HCl}$ gas over an adsorbing solution, i.e., water. Chlorosulfonic acid was added drop wise over a period of 10 min while the reaction mixture was stirred slowly in an ice bath. Let the mixture reach to room temperature and stir in an additional $2 \mathrm{~h}$. The mixture was filtered and the solid residue was washed with ether $3 \times 5$ $\mathrm{mL}$ ) and dried under vacuum. SPSA was obtained as a withe-colored solid, $3.119 \mathrm{~g}$ (95\% yields) (Scheme 1) [7]. White-colored solid, M.p. 244-248 ${ }^{\circ} \mathrm{C}$, IR (KBr) $\left(v_{\max } / \mathrm{cm}^{-1}\right): 3424,3378,3123$, 
2412, 1632, 1589, 1277, and $1143 \mathrm{~cm}^{-1} .{ }^{1} \mathrm{H}$ NMR (400 MHz, DMSO-d 6 ): $\delta 6.85(\mathrm{~d}, J=8.8 \mathrm{~Hz}, 4 \mathrm{H}$ ), $7.61(\mathrm{~d}, J=8.8 \mathrm{~Hz}, 4 \mathrm{H}), 7.95$ (s, 2H, 2OH), 10.92 (s, 2H, 2NH). ${ }^{13 C}$ NMR (100 MHz, CDCl $): \delta 146.9$, 133.7, 129.6, 118.6.

General procedure for the preparation of 3,3'-((4-chlorophenyl)methylene)bis(1H-indole)

A mixture of 4-chlorobenzaldehede ( $1 \mathrm{mmol}, 0.141 \mathrm{~g})$, and indole $(2.0 \mathrm{mmol}, 0.234 \mathrm{~g})$ and SBSA $(10 \mathrm{~mol} \%, 0.040 \mathrm{~g})$ was heated at $110^{\circ} \mathrm{C}$. The progress of the reaction was monitored by TLC $(n-$ hexane-ethyl acetate (3:1)). After completion of the reaction, the mixture was solved in hot and dry ethanol and insoluble catalyst was removed by filtration with a filter paper. The resulting crude material was purified by recrystallization from EtOH to afford pure products.

The selected spectral data

\section{3,3'-(Phenylmethylene)bis(1H-indole) (3a)}

FT-IR $(\mathrm{KBr})\left(v_{\max } / \mathrm{cm}^{-1}\right): 3482,3012,1598,1532,1465,1465,1427,1218$, and 1091. ${ }^{1} \mathrm{H}$ NMR $\left(400 \mathrm{MHz}, \mathrm{CDCl}_{3}\right): \delta 5.86(\mathrm{~s}, 1 \mathrm{H}), 6.66(\mathrm{~s}, 2 \mathrm{H}), 7.11(\mathrm{t}, J=6.9 \mathrm{~Hz}, 2 \mathrm{H}), 7.14-7.22(\mathrm{~m}, 3 \mathrm{H}), 7.28-7.31$ (m, 2H), 7.35-7.42 (m, 6H), 7.93 (br, 2H, NH).

\section{3,3'-((4-Chlorophenyl)methylene)bis(1H-indole) (3b)}

FT-IR $(\mathrm{KBr})\left(v_{\max } / \mathrm{cm}^{-1}\right): 3417,1637,1486,1455,1416,1338$, and $1092 .{ }^{1} \mathrm{H}$ NMR $(400 \mathrm{MHz}$, $\left.\mathrm{CDCl}_{3}\right): \delta 5.87(\mathrm{~s}, 1 \mathrm{H}), 6.83(\mathrm{~s}, 2 \mathrm{H}), 6.87(\mathrm{t}, J=7.5 \mathrm{~Hz}, 2 \mathrm{H}), 7.04(\mathrm{t}, J=7.5 \mathrm{~Hz}, 2 \mathrm{H}), 7.27(\mathrm{~d}, J=7.9 \mathrm{~Hz}$, 2H), $7.31(\mathrm{~d}, J=8.5 \mathrm{~Hz}, 2 \mathrm{H}), 7.39-7.33(\mathrm{~m}, 4 \mathrm{H}), 10.86(\mathrm{~s}, 2 \mathrm{H}) \mathrm{ppm}$.
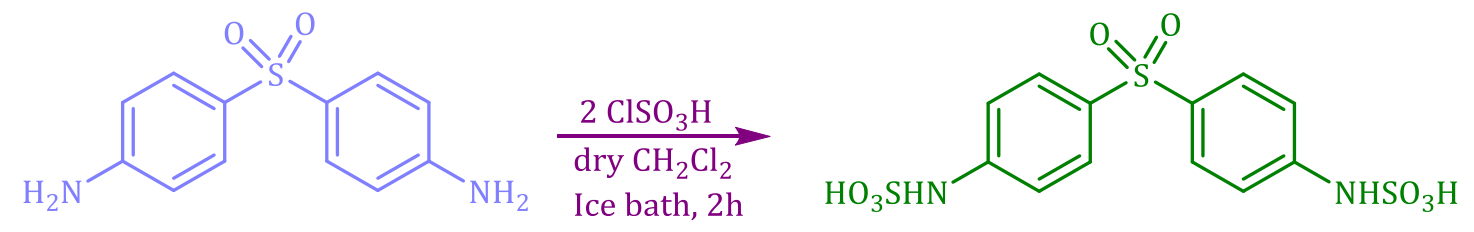

Scheme 1. Preparation of sulfonylbis(4,1-phenylene)bis(sulfamic acid) 


\section{3,3'-((2-Chlorophenyl)methylene)bis(1H-indole) (3c)}

FT-IR $(\mathrm{KBr})\left(v_{\max } / \mathrm{cm}^{-1}\right): 3414,1627,1458,1417,1338$, and $1093 .{ }^{1} \mathrm{H}$ NMR $\left(400 \mathrm{MHz}, \mathrm{CDCl}_{3}\right): \delta$ $6.34(\mathrm{~s}, 1 \mathrm{H}), 6.62(\mathrm{~s}, 2 \mathrm{H}), 7.06(\mathrm{~d}, J=8.0 \mathrm{~Hz}, 2 \mathrm{H}), 7.14-7.10(\mathrm{~m}, 1 \mathrm{H}), 7.17(\mathrm{~s}, 2 \mathrm{H}), 7.21(\mathrm{~s}, 1 \mathrm{H}), 7.23(\mathrm{~s}$, 1H), $7.38(\mathrm{~d}, J=8.1 \mathrm{~Hz}, 2 \mathrm{H}), 7.44(\mathrm{~d}, J=7.9 \mathrm{~Hz}, 2 \mathrm{H}), 7.64(\mathrm{~d}, J=7.8 \mathrm{~Hz}, 1 \mathrm{H}), 7.93(\mathrm{~s}, 2 \mathrm{H})$.

\section{3,3'-((2-Hydroxyphenyl)methylene)bis(1H-indole) (3d)}

FT-IR (KBr) $\left(v_{\max } / \mathrm{cm}^{-1}\right): 3419,2924,1620,1516,1483,1455,1420,1195$, and 1096; ${ }^{1 H}$ NMR $\left(400 \mathrm{MHz}, \mathrm{CDCl}_{3}\right): \delta 5.93(\mathrm{~s}, 1 \mathrm{H}), 6.70(\mathrm{~s}, 2 \mathrm{H}), 6.78(\mathrm{~d}, J=6.2 \mathrm{~Hz}, 1 \mathrm{H}), 6.80(\mathrm{~d}, J=3.8 \mathrm{~Hz}, 1 \mathrm{H}), 6.95(\mathrm{t}, J$ $=7.5 \mathrm{~Hz}, 2 \mathrm{H}$ ), 7.09 (d, J = 7.0 Hz, 2H), 7.13 (d, J=8.1 Hz, 2H), 7.29 (s, 1H), 7.19 (s, 2H), 7.31 (d, J = 3.4 $\mathrm{Hz}, 2 \mathrm{H}), 7.34(\mathrm{~s}, 1 \mathrm{H}), 7.96(\mathrm{~s}, 2 \mathrm{H})$.

\section{3,3'-((4-Hydroxylphenyl)methylene)bis(1H-indole) (3e)}

FT-IR $(\mathrm{KBr})\left(v_{\max } / \mathrm{cm}^{-1}\right): 3416,1617,1509,1454,1416,1338,1218,1166$, and 1095. ${ }^{1} \mathrm{H}$ NMR $\left(400 \mathrm{MHz}, \mathrm{CDCl}_{3}\right): \delta 5.70(\mathrm{~s}, 1 \mathrm{H}), 6.65(\mathrm{~d}, J=8.4 \mathrm{~Hz}, 2 \mathrm{H}), 6.77(\mathrm{~s}, 2 \mathrm{H}), 6.85(\mathrm{t}, J=7.4 \mathrm{~Hz}, 2 \mathrm{H}), 7.02(\mathrm{t}, J$ $=7.5 \mathrm{~Hz}, 2 \mathrm{H}), 7.13(\mathrm{~d}, J=8.3 \mathrm{~Hz}, 2 \mathrm{H}), 7.26(\mathrm{~d}, J=7.9 \mathrm{~Hz}, 2 \mathrm{H}), 7.33(\mathrm{~d}, J=8.1 \mathrm{~Hz}, 2 \mathrm{H}), 9.14(\mathrm{~s}, 1 \mathrm{H})$, $10.77(\mathrm{~s}, 2 \mathrm{H})$.

\section{3,3'-((3-Nitrophenyl)methylene)bis(1H-indole) (3F)}

FT-IR $(\mathrm{KBr})\left(v_{\max } / \mathrm{cm}^{-1}\right): 3411,1637,1508,1456,1417,1339$, and 1094. ${ }^{1} \mathrm{H}$ NMR (400 MHz, DMSO- $\left.d_{6}\right): \delta 6.07(\mathrm{~s}, 1 \mathrm{H}), 6.88(\mathrm{~m}, 4 \mathrm{H}), 7.06(\mathrm{t}, J=7.2 \mathrm{~Hz}, 2 \mathrm{H}), 7.30(\mathrm{~d}, J=8 \mathrm{~Hz}, 2 \mathrm{H}), 7.37(\mathrm{~d}, J=8.0 \mathrm{~Hz}$, $2 \mathrm{H}), 7.58(\mathrm{t}, J=8.4 \mathrm{~Hz}, 1 \mathrm{H}), 7.84(\mathrm{~d}, J=8.0 \mathrm{~Hz}, 1 \mathrm{H}), 8.07(\mathrm{~d}, J=8.0 \mathrm{~Hz}, 1 \mathrm{H}), 8.17(\mathrm{~s}, 1 \mathrm{H}), 10.94(\mathrm{~s}, 2 \mathrm{H})$ ppm.

\section{3,3'-((4-Nitrophenyl)methylene)bis(1H-indole) (3g)}

FT-IR (KBr) $\left(\nu_{\max } / \mathrm{cm}^{-1}\right): 3455,1637,1506,1456,1414,1341$, and 1101. ${ }^{1} \mathrm{H}$ NMR (400 MHz, $\left.\mathrm{CDCl}_{3}\right): \delta 6.04(\mathrm{~s}, 1 \mathrm{H}), 6.96-6.78(\mathrm{~m}, 4 \mathrm{H}), 7.06(\mathrm{t}, J=7.5 \mathrm{~Hz}, 2 \mathrm{H}), 7.30(\mathrm{~d}, J=7.9 \mathrm{~Hz}, 2 \mathrm{H}), 7.38(\mathrm{~d}, J=$ $8.1 \mathrm{~Hz}, 2 \mathrm{H}), 7.61(\mathrm{~d}, J=8.7 \mathrm{~Hz}, 2 \mathrm{H}), 8.15(\mathrm{~d}, J=8.7 \mathrm{~Hz}, 2 \mathrm{H}), 10.95(\mathrm{~s}, 2 \mathrm{H})$.

\section{3,3'-((4-Bromophenyl)methylene)bis(1H-indole) (3h)}

FT-IR (KBr) $\left(v_{\max } / \mathrm{cm}^{-1}\right): 3402,3058,2924,2854,1617,1457$, and 1010. ${ }^{1} \mathrm{H}$ NMR (400 MHz, $\left.\mathrm{CDCl}_{3}\right): \delta 5.91(\mathrm{~s}, 1 \mathrm{H}), 6.79(\mathrm{~d}, J=7.2 \mathrm{~Hz}, 2 \mathrm{H}), 6.87(\mathrm{t}, J=7.5 \mathrm{~Hz}, 2 \mathrm{H}), 7.07(\mathrm{t}, J=7.4 \mathrm{~Hz}, 2 \mathrm{H}), 7.28(\mathrm{~d}, J$ $=8 \mathrm{~Hz}, 2 \mathrm{H}), 7.36-7.40(\mathrm{~m}, 6 \mathrm{H}), 10.93(\mathrm{~s}, 2 \mathrm{H})$. 
3,3'-((3-Methoxyphenyl)methylene)bis(1H-indole) (3i)

FT-IR $(\mathrm{KBr})\left(v_{\max } / \mathrm{cm}^{-1}\right): 3400,3047,2964,1591$, and $1253 .{ }^{1} \mathrm{H}$ NMR $\left(400 \mathrm{MHz}, \mathrm{CDCl}_{3}\right): \delta 3.73$ $(\mathrm{s}, 3 \mathrm{H}), 5.86(\mathrm{~s}, 1 \mathrm{H}), 6.67-6.75(\mathrm{~d}, J=8.7 \mathrm{~Hz}, 2 \mathrm{H}), 6.93-7.37(\mathrm{~m}, 12 \mathrm{H}), 7.90(\mathrm{~s}, 2 \mathrm{H}, \mathrm{NH})$.

\section{3,3'-((4-Methoxyphenyl)methylene)bis(1H-indole) (3j)}

FT-IR (KBr) $\left(v_{\max } / \mathrm{cm}^{-1}\right): 3412,1636,1564,1509,1455,1417,1340,1250,1175,1095$, and 1027. ${ }_{1}^{1} \mathrm{H}$ NMR (400 MHz, $\left.\mathrm{CDCl}_{3}\right): \delta 3.77(\mathrm{~s}, 3 \mathrm{H}), 5.83(\mathrm{~s}, 1 \mathrm{H}), 6.63(\mathrm{~s}, 2 \mathrm{H}), 6.81(\mathrm{~d}, J=8.6 \mathrm{~Hz}, 2 \mathrm{H}), 7.00(\mathrm{t}, J=$ $7.3 \mathrm{~Hz}, 2 \mathrm{H}), 7.16$ (t, $J=7.4 \mathrm{~Hz}, 2 \mathrm{H}), 7.24(\mathrm{~d}, J=7.9 \mathrm{~Hz}, 2 \mathrm{H}), 7.34(\mathrm{~d}, J=8.1 \mathrm{~Hz}, 2 \mathrm{H}), 7.38$ (d, $J=8.0 \mathrm{~Hz}$, 2H), $7.90(\mathrm{~s}, 2 \mathrm{H})$.

\section{3,3'-((4-Methylphenyl)methylene)bis(1H-indole) (3k)}

FT-IR (KBr) $\left(\nu_{\max } / \mathrm{cm}^{-1}\right): 3421,1635,1512,1454,1416,1339$, and 1095. ${ }^{1} \mathrm{H}$ NMR (400 MHz, $\left.\mathrm{CDCl}_{3}\right): \delta 2.31(\mathrm{~s}, 3 \mathrm{H}), 5.85(\mathrm{~s}, 1 \mathrm{H}), 6.66(\mathrm{~s}, 2 \mathrm{H}), 7.00(\mathrm{t}, J=7.5 \mathrm{~Hz}, 2 \mathrm{H}), 7.08(\mathrm{~d}, J=7.7 \mathrm{~Hz}, 2 \mathrm{H}), 7.16(\mathrm{t}$, $J=7.5 \mathrm{~Hz}, 2 \mathrm{H}$ ), 7.22 (d, $J=7.9 \mathrm{~Hz}, 2 \mathrm{H}), 7.35$ (d, $J=8.1 \mathrm{~Hz}, 2 \mathrm{H}), 7.39$ (d, $J=8.0 \mathrm{~Hz}, 2 \mathrm{H}), 7.91(\mathrm{~s}, 2 \mathrm{H})$.

\section{3,3'-((4-Nitrophenyl)methylene)bis(2-methyl-1H-indole) (3l)}

FT-IR (KBr) $\left(v_{\max } / \mathrm{cm}^{-1}\right): 3418,1647,1512,1449,1410$, and 1335. ${ }^{1} \mathrm{H}$ NMR (400 MHz, DMSO- $d_{6}$ ): $\delta 3.75(\mathrm{~s}, 6 \mathrm{H}), 6.05(\mathrm{~s}, 1 \mathrm{H}), 6.62(\mathrm{~s}, 2 \mathrm{H}), 7.08(\mathrm{t}, J=7.3 \mathrm{~Hz}, 2 \mathrm{H}), 7.29(\mathrm{t}, J=7.3 \mathrm{~Hz}, 2 \mathrm{H}), 7.39(\mathrm{t}, J=8.6$ $\mathrm{Hz}, 4 \mathrm{H}), 7.56(\mathrm{~d}, J=8.6 \mathrm{~Hz}, 2 \mathrm{H}), 8.18(\mathrm{~d}, J=8.6 \mathrm{~Hz}, 2 \mathrm{H})$.

\section{4-(Bis(2-methyl-1H-indol-3-yl)methyl)phenol (3m)}

FT-IR $(\mathrm{KBr})\left(v_{\max } / \mathrm{cm}^{-1}\right): 3423,1611,1516,1438,1421,1341$, and 1082. ${ }^{1} \mathrm{H}$ NMR $(400 \mathrm{MHz}$, $\left.\mathrm{CDCl}_{3}\right): \delta 1.85(\mathrm{~s}, 6 \mathrm{H}), 5.64(\mathrm{~s}, 1 \mathrm{H}), 6.50(\mathrm{~m}, 4 \mathrm{H}), 6.71(\mathrm{~m}, 4 \mathrm{H}), 7.01(\mathrm{~d}, J=7.5 \mathrm{~Hz}, 2 \mathrm{H}), 7.17(\mathrm{~d}, J=7.5$ $\mathrm{Hz}, 2 \mathrm{H}), 8.62(\mathrm{~s}, \mathrm{H}), 9.65(\mathrm{~s}, 2 \mathrm{H})$.

\section{4-(Bis(2-methyl-1H-indol-3-yl)methyl)-N,N-dimethylaniline (3n)}

FT-IR (KBr) $\left(v_{\max } / \mathrm{cm}^{-1}\right): 3410,3050,1510,1455,1245,1135$, and 1020. ${ }^{1} \mathrm{H}$ NMR (400 MHz, $\left.\mathrm{CDCl}_{3}\right): \delta 2.08(\mathrm{~s}, 6 \mathrm{H}), 3.71(\mathrm{~s}, 3 \mathrm{H}), 3.85(\mathrm{~s}, 3 \mathrm{H}), 5.96(\mathrm{~s}, 1 \mathrm{H}), 6.75(\mathrm{~s}, 2 \mathrm{H}), 6.85(\mathrm{~m}, 3 \mathrm{H}), 7.03(\mathrm{t}, J=6.4$ $\mathrm{Hz}, 4 \mathrm{H}), 7.24(\mathrm{t}, J=7.6 \mathrm{~Hz}, 2 \mathrm{H}), 7.77(\mathrm{~s}, 2 \mathrm{H})$.

\section{Results and discussion}

In order to optimize the reaction conditions, we have used of SPSA as a catalyst for the condensation of indole with 4-chlorobenzaldehede at various conditions such as different amounts 
of catalyst, temperature, solvent and solvent-free conditions (Table 1). A highly dense orange mixture was prepared with down yield after a long time. Hence, we investigated the catalytic effect of SPSA for the same reactions. We noticed that when the reaction is carried out in the presence of SPSA catalyst, in addition to a significant reduction in reaction time, the product yield became more. These studies showed that the best result was obtained by carrying out the reaction of 4chlorobenaldehyde (1 mmol) with indole $(2 \mathrm{mmol})$ in the presence of $10 \mathrm{~mol} \%$ of SBSA at $110{ }^{\circ} \mathrm{C}$ for 30 min under solvent-free conditions (Table 1, Entry 9).

After optimization of the reaction conditions (Table 1, Entry 9) and in order to show the extent of the application of the method, various types of aldehydes and indoles were subjected to the similar reaction under the determined conditions (Scheme 2). As shown in Table 2, the corresponding compounds were obtained during relatively short reaction times in high yields.

Table 1. Optimization of the amount of the catalyst, temperature and solvent in the synthesis of bis(indolyl) methane derivative of 4-chlorobenzaldehyde

\begin{tabular}{cccccc} 
Entry & Amount of catalyst $(\mathrm{mol} \%)$ & Solvent & Temp. $\left({ }^{\circ} \mathrm{C}\right)$ & Time $(\mathrm{min})$ & ${\text { Yield }(\%)^{\mathrm{a}}}$ \\
1 & - & Solvent-free & 100 & 120 & Trace \\
2 & - & EtOH & Reflux & 120 & Trace \\
3 & 5 & Solvent-free & 100 & 60 & 72 \\
4 & 5 & EtOH & Reflux & 100 & 65 \\
5 & 5 & $\mathrm{H}_{2} \mathrm{O}$ & Reflux & 100 & 40 \\
6 & 5 & $\mathrm{CH}_{3} \mathrm{CN}$ & Reflux & 100 & Trace \\
7 & 10 & Solvent-free & 80 & 50 & 85 \\
8 & 10 & Solvent-free & 100 & 45 & 93 \\
9 & 10 & Solvent-free & 110 & 30 & 97 \\
10 & 10 & Solvent-free & 120 & 30 & 95 \\
\hline
\end{tabular}

${ }^{a}$ Isolated yield

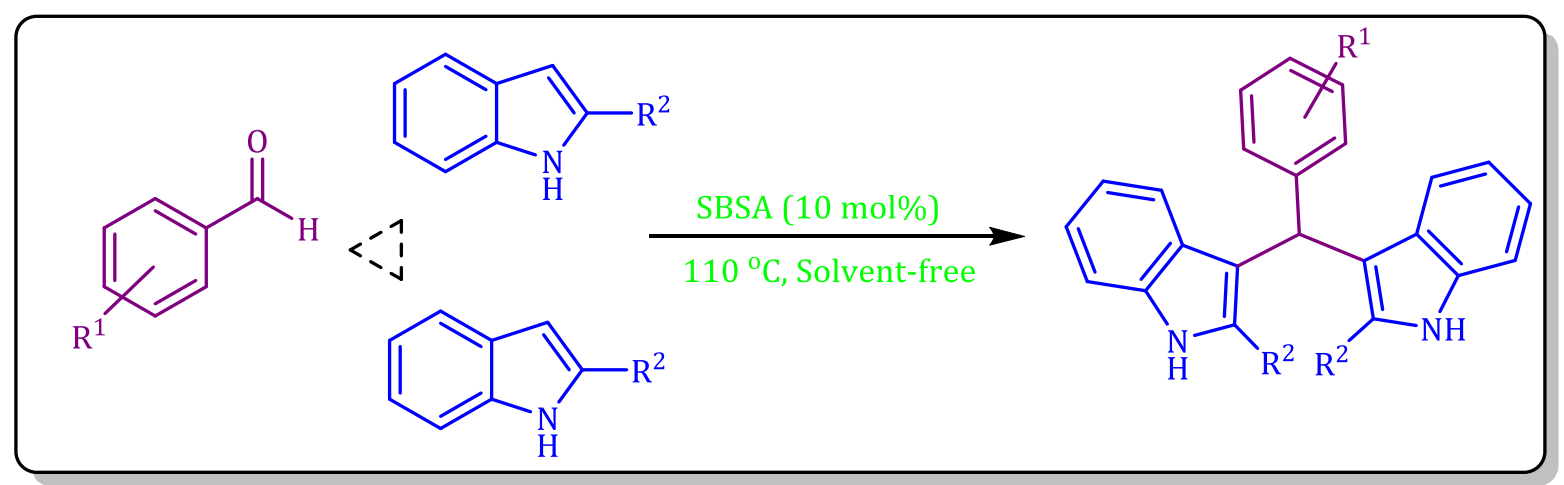

Scheme 2. Synthesis of bis(indolyl)methane derivatives 
Table 2. Synthesis of bis(indolyl)methanes in the absence of solvent ${ }^{\text {a }}$

\begin{tabular}{cccccc} 
Entry & Aldehyde & $\mathrm{R}^{2}$ & Product & Time (min) & Yield (\%) [ref.] \\
1 & $\mathrm{C}_{6} \mathrm{H}_{4} \mathrm{CHO}$ & $\mathrm{H}$ & $3 \mathrm{a}$ & 40 & $92[32]$ \\
2 & $4-\mathrm{ClC}_{6} \mathrm{H}_{4} \mathrm{CHO}$ & $\mathrm{H}$ & $3 \mathrm{~b}$ & 30 & $97[33]$ \\
3 & $2-\mathrm{ClC}_{6} \mathrm{H}_{4} \mathrm{CHO}$ & $\mathrm{H}$ & $3 \mathrm{c}$ & 35 & $95[32]$ \\
4 & $2-\mathrm{OHC}_{6} \mathrm{H}_{4} \mathrm{CHO}$ & $\mathrm{H}$ & $3 \mathrm{~d}$ & 50 & $88[34]$ \\
5 & $4-\mathrm{OHC}_{6} \mathrm{H}_{4} \mathrm{CHO}$ & $\mathrm{H}$ & $3 \mathrm{e}$ & 50 & $87[33]$ \\
6 & $3-\mathrm{NO}_{2} \mathrm{C}_{6} \mathrm{H}_{4} \mathrm{CHO}$ & $\mathrm{H}$ & $3 \mathrm{f}$ & 35 & $95[33]$ \\
7 & $4-\mathrm{NO}_{2} \mathrm{C}_{6} \mathrm{H}_{4} \mathrm{CHO}$ & $\mathrm{H}$ & $3 \mathrm{~g}$ & 40 & $93[35]$ \\
8 & $4-\mathrm{BrC}_{6} \mathrm{H}_{4} \mathrm{CHO}$ & $\mathrm{H}$ & $3 \mathrm{~h}$ & 45 & $90[32]$ \\
9 & $3-\mathrm{OCH}_{3} \mathrm{C}_{6} \mathrm{H}_{4} \mathrm{CHO}$ & $\mathrm{H}$ & $3 \mathrm{i}$ & 50 & $88[36]$ \\
10 & $4-\mathrm{OCH}_{3} \mathrm{C}_{6} \mathrm{H}_{4} \mathrm{CHO}$ & $\mathrm{H}$ & $3 \mathrm{j}$ & 55 & $97[32]$ \\
11 & $4-\mathrm{CH}_{3} \mathrm{C}_{6} \mathrm{H}_{4} \mathrm{CHO}$ & $\mathrm{H}$ & $3 \mathrm{k}$ & 50 & $90[37]$ \\
12 & $4-\mathrm{NO}_{2} \mathrm{C}_{6} \mathrm{H}_{4} \mathrm{CHO}$ & $\mathrm{Me}$ & $3 \mathrm{l}$ & 45 & $91[37]$ \\
13 & $4-\mathrm{HOC}_{6} \mathrm{H}_{4} \mathrm{CHO}$ & $\mathrm{Me}$ & $3 \mathrm{~m}$ & 60 & $88[37]$ \\
14 & $3,4-\left(\mathrm{MeO}_{2} \mathrm{C}_{6} \mathrm{H}_{3} \mathrm{CHO}\right.$ & $\mathrm{Me}$ & $3 \mathrm{n}$ & 50 & $89[37]$ \\
\hline
\end{tabular}

${ }^{\mathrm{a}}$ Isolated yield

A reasonable mechanism for the preparation of bis(indole)methanes is shown in Scheme 3. in the first stage, the aldehyde is activated by using $\mathrm{H}^{+}$catalyst. Reaction of intermediate I with indole led to the formation of intermediate II and loss of $\mathrm{H}_{2} \mathrm{O}$ from II to afford IV. In the following step, the desired bis-indole was obtained from the reaction of IV and other indole in the presence of catalyst.

The ability of the current method is shown through comparison with the results of other catalysts in the literature (Table 3). The reaction of the preparation of bis(indole)methane was accelerated in the presence of sulfonylbis(1,4-phenylene)bissulfamic acid (SPSA) and the reaction was completed in short time. Our discussed catalyst (SPSA) can with of the release two acidic hydrogen from both of the active positions, causes the more increase in yield and reduce the reaction times.

Table 3. Compared efficiency of various catalysts in preparation of bis(indolyl) methane of 4$\mathrm{ClC}_{6} \mathrm{H}_{4} \mathrm{CHO}$

\begin{tabular}{|c|c|c|c|c|}
\hline Entry & Catalyst & Conditions & Time (h) & Yield (\%)[Ref.] \\
\hline 1 & TBATB & $\mathrm{MeOH}$, r.t. & 1.5 & 85 [15] \\
\hline 2 & $\mathrm{M}\left(\mathrm{HSO}_{4}\right) \mathrm{x}$ & EtOH, r.t. & $2.5-7.5$ & $91[16]$ \\
\hline 3 & $\mathrm{~V}\left(\mathrm{HSO}_{4}\right)_{3}$ & Solvent-free, $80^{\circ} \mathrm{C}$ & 0.66 & 85 [33] \\
\hline 4 & ВТРТВ & Solvent-free, $90^{\circ} \mathrm{C}$ & 0.13 & 80 [37] \\
\hline 5 & $\mathrm{P}_{2} \mathrm{O}_{5} / \mathrm{SiO}_{2}$ & Solvent-free, r.t. & 0.5 & 92 [38] \\
\hline 6 & {$[\mathrm{hmim}][\mathrm{HSO} 4]$} & EtOH, r.t. & 1 & 93 [39] \\
\hline 7 & ILIS-SO ${ }_{2} \mathrm{Cl}$ & $\mathrm{MeCN}$, r.t. & 5.5 & $93[40]$ \\
\hline 8 & Zeokarb-225 & $\mathrm{CH}_{3} \mathrm{CN}$, r.t. & 8.5 & $70[41]$ \\
\hline 9 & SQ & $\mathrm{H}_{2} \mathrm{O} /$ r.t. & $2-4$ & $82[35]$ \\
\hline 10 & SPSA & Solvent-free, $110^{\circ} \mathrm{C}$ & 0.5 & 97 [this work] \\
\hline
\end{tabular}




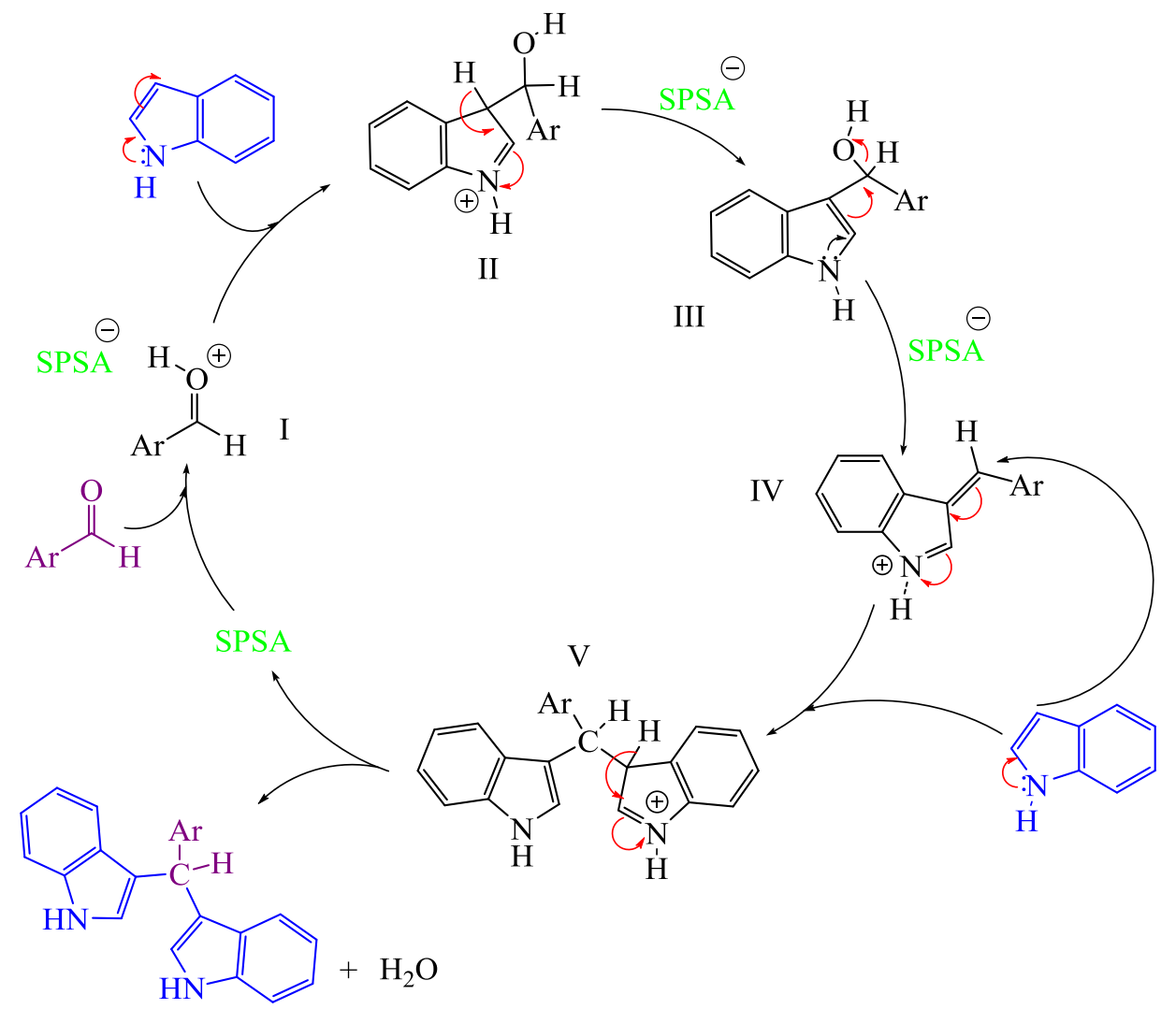

Scheme 3. The reasonable mechanism in preparation of bis(indolyl)methane

\section{Conclusion}

In conclusion, we have described a simple and efficient method for the preparation of bis(indole)methane derivatives catalyzed using SPSA under solvent-free conditions. The procedure offers several advantages such as relatively short reaction times, high yields of the products, simple experimental procedure, easy and clean work-up methode, solvent-free conditions and as ease of the preparation and simple recyclability of the SPSA, Which makes this method as a suitable route compared with available methods for the synthesis of bis(indole)methanes.

\section{References}

[1]. Armstrong R.W., Combs A.P., Tempest P.A., Brown S.D., Keating T.A. Acc. Chem. Res., 1996,

29:123

[2]. Ibrahim Y.A., Behbehani H., Ibrahim M.R. Tetrahedron Lett., 2002, 43:4207

[3]. Tabrizian E., Amoozadeh A., Rahmani S., Imanifar E., Azhari S., Malmir M. Chin. Chem. Lett., 2015, 26:1278

[4]. Khaksar S., Tajbakhsh M., Gholami M. C. R. Chimie., 2014, 17:30 
[5]. Saeidian H., Moradnia F. Iran. Chem. Commun., 2017, 5:252

[6]. Kale S.R., Kahandal S.S., Burange A.S., Gawande M.B., Jayaram R.V. Catal. Sci. Technol., 2013, 3:2050

[7]. Mahmoodi, N.O., Khazaei Z., Zeydi M.M. J. Iran. Chem. Soc., 2017, 4:1889

[8]. Endo T., Tsuda M., Fromont J., Kobayashi J., Hyrtinadine A. J. Nat. Prod., 2007, 70:423

[9]. Kalla R.M.N., Khalivulla S.I., Rasheed S., Fakurazi S., Arulselvan P., Lasekan O., Abas F. Int. J. Mol. Sci., 2013, 14:1843

[10]. Kamal A., Khan M.N.A., Reddy K.S., Srikanth Y.V.V., Ahmed S.K., Kumar K.P., Murthy U.S.N. J. Enzyme. Inhib. Med. Chem., 2009, 24:559

[11]. Benabadji S.H., Wen R., Zheng J., Dong X., Yuan S. Acta. Pharmacol. Sinica., 2004, 25:666

[12]. Sujatha K., Perumal P.T., Muralidharan D., Rajendran M. Indian J. Chem., 2009, 48:267

[13]. Karthik M., Tripathi A.K., Gupta N.M., Palanichamy M., Murugesan V. Catal. Commun., 2004, $\mathbf{5 : 3 7 1}$

[14]. Pore D.M., Desai U.V., Thopate T.S., Wadgaonkar P.P. Arkivoc, 2006, 12:75

[15]. Lin X.F., Cui S.L., Wang Y.G. Synth. Commun., 2006, 36:3153

[16]. Niknam K., Zolfigol M.A., Sadabadi T., Nejati A. J. Iran. Chem. Soc., 2006, 3:318

[17]. Reddy B.M., Sreekanth P.M., Lakshmanan P. J. Mol. Catal. A Chem., 2005, 237:93

[18]. Maiti A.K., Bhattacharyya P. J. Chem. Res., 1997, s:424

[19]. Deb M.L., Bhuyan P.J. Tetrahedron Lett., 2006, 47:1441

[20]. Sharma G.V.M., Reddy J.J., Lakshmi P.S., Krishna P.R. Tetrahedron Lett., 2004, 45:7729

[21]. Karthik M., Tripathi A.K., Gupta N.M., Palanichamy M., Murugesan V. Catal Commun., 2004, 5:371

[22]. Firouzabadi H., Iranpoor N., Jafari A.A. J. Mol. Catal. A Chem., 2005, 244:168

[23]. Pradhan P.K., Dey S., Giri V.S., Jaisankar P. Synthesis, 2005, 37:1779

[24]. Azizi N., Torkian L., Saidi M.R. J. Mol. Catal. A Chem., 2007, 275:109

[25]. Hosseini-Sarvari M. Acta Chim. Slovenica, 2007, 54:354

[26]. Mehrazma S., Azizi N., Saidi M.R. Lett. Org. Chem., 2006, 3:161

[27]. Firouzabadi H., Iranpoor N., Jafarpour M., Ghaderi A. J. Mol. Catal. A Chem., 2006, 253:249

[28]. Kamble V.T., Bandgar B.P., Bavikar S.N. Chin. J. Chem., 2007, 25;13

[29]. Kamble V.T., Kadam K.R., Joshi N.S., Muley D.B. Catal. Commun., 2007, 8:498

[30]. Koshima H., Matsuaka W. J. Heterocycl. Chem., 2002, 39:1089

[31]. Khodaei M.M., Mohammadpoor-Baltork I., Memarian H.R., Khosropour A.R., Nikofar K., Ghanbary P. J. Heterocycl. Chem., 2008, 45:1 
[32]. Ghorbani-Vaghei R., Veisi H., Keypour H., Dehghani-Firouzabadi A. A. Mol. Divers, 2010, 14:87

[33]. Shirini F., Yahyazadeh A., Abedini M., Imani Langroodi D. Bull. Korean Chem. Soc., 2010, 31:

1715

[34]. Rekha M., Manjunath H.R., Nagaraju N. J. Indust. Engin. Chem., 2013, 19:337

[35]. Azizi N., Gholibeghlo E., Manocheri Z. ScientiaIranica C, 2012, 19:574

[36]. Jhansi Rani V., Veena Vani K., Venkata Rao C. Synth. Commun., 2012, 42:2048

[37]. Shirini F., Safarpoor Langroodi M., Abedini M. Chin. Chem. Lett., 2010, 21:1342

[38]. Hasaninejad A., Zare A., Sharghi H., Niknam K., Shekouhy M. Arkivoc, 2007, xiv:39

[39]. Gong G.D., Shun-Jun J.I., Zhao-Qin J., Min-Feng Z., Teck-Peng L. Synlett, 2005, 959

[40]. Hagiwara H., Sekifuji M., Hoshi T., Qiao K., Yokoyamac C. Synlett, 2007, 1320

[41]. Magesh C.J., Nagarajan R., Karthink M., Perumal P.T. Appl. Catal. A: General, 2004, 266:1

How to cite this manuscript: Masoud Mohammadi Zeydi*, Nosrat 0 Allah Mahmoodi, Ghorban Ardeshiri Terogeni. Sulfonylbis(1,4-phenylene)bissulfamic acid (SPSA): Introduction of an efficient and reusable catalyst for the synthesis of bis(indolyl)methanes. Asian Journal of Green Chemistry, 2017, 1, 78-88. DOI:10.22631/ajgc.2017.95432.1013 